\title{
On intuitionistic fuzzy version of Zadeh's extension principle
}

\author{
Selami Bayeğ $\breve{g}^{1}$ and Raziye Mert ${ }^{2}$ \\ ${ }^{1}$ Industrial Engineering Department, \\ University of Turkish Aeronautical Association, Ankara, Turkey \\ e-mail: sbayeg@thk.edu.tr \\ ${ }^{2}$ Department of Software Engineering, \\ Adana Alparslan Turkes Science and Technology University, Adana, Turkey \\ e-mail: rmerteatu.edu.tr
}

\begin{abstract}
In this paper, by using $\alpha$ - and $\beta$-cuts approach and the intuitionistic fuzzy Zadeh's extension principle, we have proved a result which reveals that the $\alpha$ - and $\beta$-cuts of an intuitionistic fuzzy number obtained by the intuitionistic fuzzy Zadeh's extension principle coincide with the images of the $\alpha$ - and $\beta$-cuts by the crisp function. Then we have given a corollary about monotonicity of the extension principle. Finally, we have extended these results to $I F_{N}(\mathbb{R}) \times I F_{N}(\mathbb{R})$.
\end{abstract}

Keywords: Intuitionistic fuzzy sets, Intuitionistic fuzzy Zadeh's extension principle, Zadeh's extension principle.

2020 Mathematics Subject Classification: 94D05, 26E50.

\section{Introduction}

Fuzzy set theory was introduced by L. A. Zadeh in 1965 to explain vagueness in scientific models. In a fuzzy set, every element is accompanied with a function $\mu(x): X \rightarrow[0,1]$, called membership function. Hence, value of $1-\mu$ is the non-membership degree of an element and the sum of these two values is 1 for any element. However, because of inaccuracy in a data set or 
incomplete information in real world models, there can be vagueness about the non-membership of the element, too. That is why some generalizations of fuzzy set theory were developed. One of these is the intuitionistic fuzzy set theory which was introduced by Atanassov [3]. He introduced the idea of intuitionistic fuzzy sets in 1983. In this novel idea, every element in a set is not given only with a membership function $\mu(x): X \rightarrow[0,1]$ but also with a non-membership function $\nu(x): X \rightarrow[0,1]$ such that the sum of both is less than or equal to 1 .

The Zadeh's extension principle, which plays an important role in fuzzy set theory, is an essential principle to extend the classical mathematical tools into fuzzy environment [8]. There are many applications of this principle $[1,5,7,8,11]$. Two of the most important ones are: it enables us to fuzzify the classical arithmetic and binary logic operations and it enables us to produce a fuzzy mapping from a classical mapping. Because of these main applications, intuitionistic fuzzy versions of this principle were proposed $[4,9]$. With the help of this principle, one can construct the image of an intuitionistic fuzzy mapping and proceed with arithmetic operations. Besides, the intuitionistic fuzzy Zadeh's extension principle enables us to intuitionistically fuzzify some important mathematical tools based on classical set theory.

In this paper, we have aimed to extend the theorems in $[6,10]$. Firstly, we have shown that the $\alpha$ - and $\beta$-cuts of the image of a crisp function obtained by the intuitionistic fuzzy Zadeh's extension principle coincide with the $\alpha$ - and $\beta$-cuts of an intuitionistic fuzzy number. Then we have given a corollary about monotonicity of the extension principle. Finally, we have extended these results to

$$
I F_{N}(\mathbb{R}) \times I F_{N}(\mathbb{R})
$$

\section{Preliminaries}

Definition 2.1 ([3]). Let $X$ be the universe of discourse. An intuitionistic fuzzy set (IFS) in $X$ is a set of the form

$$
\tilde{A}^{i}=\left\{\left(x, \mu_{\tilde{A}^{i}}(x), \nu_{\tilde{A}^{i}}(x)\right): x \in X\right\},
$$

where the functions $\mu_{\tilde{A}^{i}}: X \rightarrow[0,1]$ and $\nu_{\tilde{A}^{i}}: X \rightarrow[0,1]$ are membership and non-membership, functions respectively, such that for every $x \in X, 0 \leq \mu_{\tilde{A}^{i}}(x)+\nu_{\tilde{A}^{i}}(x) \leq 1$.

$I F(X)$ will be used to denote the set of all intuitionistic fuzzy sets in $X$.

Definition 2.2 ([1]). The $\alpha$-cut of $\tilde{A}^{i} \in I F\left(\mathbb{R}^{n}\right)$ is defined as:

$$
A(\alpha)=\left\{x: x \in \mathbb{R}^{n}, \mu_{\tilde{A}^{i}}(x) \geq \alpha\right\},
$$

for $0<\alpha \leq 1$, and

$$
A(0)=c l\left(\bigcup_{\alpha \in(0,1]} A(\alpha)\right),
$$

for $\alpha=0$. 
Definition 2.3 ([1]). The $\beta$-cut of $\tilde{A}^{i} \in I F\left(\mathbb{R}^{n}\right)$ is defined as:

$$
A^{*}(\beta)=\left\{x: x \in \mathbb{R}^{n}, \nu_{\tilde{A}^{i}}(x) \leq \beta\right\},
$$

for $0<\beta \leq 1$, and

$$
A^{*}(1)=\operatorname{cl}\left(\bigcup_{\beta \in[0,1)} A^{*}(\beta)\right),
$$

for $\beta=0$.

Definition 2.4 ([1]). If $\tilde{A}^{i} \in I F\left(\mathbb{R}^{n}\right)$ satisfies the following conditions, then it is called an intuitionistic fuzzy number in $\mathbb{R}^{n}$ :

1. $\tilde{A}^{i}$ is a normal set, i.e., $\exists x_{0} \in \mathbb{R}^{n}$ such that $\mu_{\tilde{A}^{i}}\left(x_{0}\right)=1$ (hence, $\nu_{\tilde{A}^{i}}\left(x_{0}\right)=0$ ).

2. $A(0)$ and $A^{*}(1)$ are bounded sets in $\mathbb{R}^{n}$.

3. $\mu_{\tilde{A}^{i}}: \mathbb{R}^{n} \rightarrow[0,1]$ is upper semi-continuous: $\forall k \in[0,1]$, the set $\left\{x: x \in \mathbb{R}^{n}, \mu_{\tilde{A}^{i}}(x)<k\right\}$ is open.

4. $\nu_{\tilde{A}^{i}}: \mathbb{R}^{n} \rightarrow[0,1]$ is lower semi-continuous: $\forall k \in[0,1]$, the set $\left\{x: x \in \mathbb{R}^{n}, \nu_{\tilde{A}^{i}}(x)>k\right\}$ is open.

5. The membership function $\mu_{\tilde{A}^{i}}$ is quasi-concave:

$$
\mu_{\tilde{A}^{i}}(\lambda x+(1-\lambda) y) \geq \min \left\{\mu_{\tilde{A}^{i}}(x), \mu_{\tilde{A}^{i}}(y)\right\}, \forall x, y \in \mathbb{R}^{n}, \lambda \in[0,1] .
$$

6. The non-membership function $\nu_{\tilde{A}^{i}}$ is quasi-convex:

$$
\nu_{\tilde{A}^{i}}(\lambda x+(1-\lambda) y) \leq \max \left\{\nu_{\tilde{A}^{i}}(x), \nu_{\tilde{A}^{i}}(y)\right\}, \forall x, y \in \mathbb{R}^{n}, \lambda \in[0,1] .
$$

We will denote the set of all intuitionistic fuzzy numbers of $\mathbb{R}^{n}$ by $I F_{N}\left(\mathbb{R}^{n}\right)$.

Theorem 2.5 ([2]). Let $\bar{A}^{i} \in I F_{N}\left(\mathbb{R}^{n}\right)$ and $\alpha, \beta \in[0,1]$ be such that its $\alpha$-and $\beta$-cuts given by $A(\alpha)=\left\{x: x \in \mathbb{R}^{n}, \mu_{\tilde{A}^{i}}(x) \geq \alpha\right\}$ and $A^{*}(\beta)=\left\{x: x \in \mathbb{R}^{n}, \nu_{\tilde{A}^{i}}(x) \leq \beta\right\}$. Then the following hold:

1. $A(\alpha)$ and $A^{*}(\beta)$ are non-empty compact and convex sets in $\mathbb{R}^{n}$.

2. If $0 \leq \alpha_{1} \leq \alpha_{2} \leq 1$, then $A\left(\alpha_{2}\right) \subseteq A\left(\alpha_{1}\right)$.

3. If $0 \leq \beta_{1} \leq \beta_{2} \leq 1$, then $A^{*}\left(\beta_{1}\right) \subseteq A^{*}\left(\beta_{2}\right)$.

4. If $\left(\alpha_{n}\right)$ is a non-decreasing sequence converging to $\alpha$, then

$$
\bigcap_{n=1}^{\infty} A\left(\alpha_{n}\right)=A(\alpha) \text {. }
$$

5. If $\left(\beta_{n}\right)$ is a non-increasing sequence converging to $\beta$, then

$$
\bigcap_{n=1}^{\infty} A^{*}\left(\beta_{n}\right)=A^{*}(\beta) .
$$


6. If $\left(\alpha_{n}\right)$ is a non-increasing sequence converging to 0 , then

$$
c l\left(\bigcup_{n=1}^{\infty} A\left(\alpha_{n}\right)\right)=A(0) .
$$

7. If $\left(\beta_{n}\right)$ is a non-decreasing sequence converging to 1 , then

$$
\operatorname{cl}\left(\bigcup_{n=1}^{\infty} A^{*}\left(\beta_{n}\right)\right)=A^{*}(1) .
$$

Now, we will give the Zadeh's extension principle in the case of intuitionistic fuzzy sets.

Definition 2.6 ([4]). Assume that $X$ and $Y$ are classical sets and $f: X \rightarrow Y$ is a function. If $\tilde{A}^{i} \in I F(X)$, then $f\left(\tilde{A}^{i}\right)$ is an intuitionistic fuzzy set in $Y$ such that:

$$
\begin{aligned}
& \mu_{f\left(\tilde{A}^{i}\right)}(y)=\left\{\begin{array}{cc}
\sup \left\{\mu_{\tilde{A}^{i}}(x): f(x)=y\right\}, & y \in f(X) \\
0, & y \notin f(X)
\end{array},\right. \\
& \nu_{f\left(\tilde{A}^{i}\right)}(y)=\left\{\begin{array}{cc}
\inf \left\{\nu_{\tilde{A}^{i}}(x): f(x)=y\right\}, & y \in f(X) \\
1, & y \notin f(X)
\end{array} .\right.
\end{aligned}
$$

for every $y \in Y$.

\section{Main results}

Theorem 3.1. Given a continuous function $f: \mathbb{R} \rightarrow \mathbb{R}$, it can be extended to an intuitionistic fuzzy function $\tilde{f}^{i}: I F_{N}(\mathbb{R}) \rightarrow I F_{N}(\mathbb{R})$ such that for $\tilde{A}^{i} \in I F_{N}(\mathbb{R})$ we can determine $\tilde{B}^{i}=\tilde{f}^{i}\left(\tilde{A}^{i}\right) \in I F_{N}(\mathbb{R})$ by its $\alpha$ - and $\beta$-cuts $B(\alpha)=\tilde{f}^{i}(A(\alpha))$ and $B^{*}(\beta)=\tilde{f}^{i}\left(A^{*}(\beta)\right)$, $\forall \alpha, \beta \in[0,1]$, i.e., we have $B(\alpha)=\left[B_{1}(\alpha), B_{2}(\alpha)\right]$ and $\left.B^{*}(\beta)=\left[B_{1}^{*}(\beta), B_{2}^{*}(\beta)\right)\right]$, where

$$
\begin{aligned}
& B_{1}(\alpha)=\inf \{f(x): x \in A(\alpha)\} \\
& B_{2}(\alpha)=\sup \{f(x): x \in A(\alpha)\}, \\
& B_{1}^{*}(\beta)=\inf \left\{f(x): x \in A^{*}(\beta)\right\} \\
& B_{2}^{*}(\beta)=\sup \left\{f(x): x \in A^{*}(\beta)\right\},
\end{aligned}
$$

$\forall \alpha, \beta \in[0,1]$

Proof. We will first prove that if $A(\alpha), A^{*}(\beta)$ and $B(\alpha), B^{*}(\beta)$ are $\alpha$ - and $\beta$-cuts of the intuitionistic fuzzy numbers $\tilde{A}^{i}$ and $\tilde{B}^{i}=\tilde{f}^{i}\left(\tilde{A}^{i}\right)$, respectively, then $B(\alpha)=f(A(\alpha))$ and $B^{*}(\beta)=f\left(A^{*}(\beta)\right)$. By [6] we already know that $B(\alpha)=f(A(\alpha))$. Let us prove that $B^{*}(\beta)=f\left(A^{*}(\beta)\right)$.

If $f^{-1}(y)=\emptyset$, then $\nu_{\tilde{B}^{i}}(y)=1$ and there is nothing to do. Assume that $f^{-1}(y) \neq \emptyset$. Then, by Definition 2.6, we have

$$
\nu_{\tilde{B}^{i}}(y)=\inf \left\{\nu_{\tilde{A}^{i}}(x): x \in X, f(x)=y\right\}
$$


If $x \in A^{*}(\beta)$, then $\nu_{\tilde{A}^{i}}(x) \leq \beta$ and it implies also that $\nu_{\tilde{B}^{i}}(y) \leq \beta$ and so $y=f(x) \in B^{*}(\beta)$, i.e., $f\left(A^{*}(\beta)\right) \subseteq B^{*}(\beta)$. On the other hand, if $\nu_{\tilde{B}^{i}}(y) \leq \beta$, then for each $\varepsilon=\frac{1}{n}, n \in \mathbb{N}$, there exists $x_{n} \in f^{-1}(y)$ such that

$$
\nu_{\tilde{A}^{i}}\left(x_{n}\right)<\nu_{\tilde{B}^{i}}(y)+\frac{1}{n} .
$$

Let $\nu_{\tilde{A}^{i}}\left(x_{n}\right) \leq \beta_{n}$. Since $\left\{x_{n}\right\}$ is a bounded sequence and since $x_{n} \in A^{*}(1)$ and $A^{*}(1)$ is compact, it has a convergent subsequence $\left\{x_{n_{k}}\right\}$. Let $x=\lim _{k \rightarrow \infty} x_{n_{k}}$. Since $\nu_{\tilde{A}^{i}}$ is lower-semi continuous, it follows that $\lim _{k \rightarrow \infty} \nu_{\tilde{A}^{i}}\left(x_{n_{k}}\right) \geq \nu_{\tilde{A}^{i}}(x)$. Hence, from (1) and the fact that $\nu_{\tilde{B}^{i}}(y) \leq$ $\beta$, we have $\nu_{\tilde{A}^{i}}(x) \leq \beta$ also. And since $f\left(x_{n_{k}}\right)=y$ and $f$ is continuous, $f(x)=y$. Then, $B^{*}(\beta) \subseteq f\left(A^{*}(\beta)\right)$. Finally, we have $B^{*}(\beta)=f\left(A^{*}(\beta)\right)$. So, if $\left.B^{*}(\beta)=\left[B_{1}^{*}(\beta), B_{2}^{*}(\beta)\right)\right]$, we obtain

$$
\begin{aligned}
& B_{1}^{*}(\beta)=\inf \left\{f(x): x \in A^{*}(\beta)\right\}, \\
& B_{2}^{*}(\beta)=\sup \left\{f(x): x \in A^{*}(\beta)\right\} .
\end{aligned}
$$

Now, we will prove that if $A^{*}(\beta)$ are $\beta$-cuts of an intuitionistic fuzzy number $\tilde{A}^{i}$, then $B^{*}(\beta)=f\left(A^{*}(\beta)\right)$ are $\beta$-cuts of an intuitionistic fuzzy number $\tilde{B}^{i}=\tilde{f}^{i}\left(\tilde{A}^{i}\right)$ by using the intuitionistic fuzzy version of the characterization theorem [2]. First, we note that since $A^{*}(\beta)$ are compact convex intervals in $\mathbb{R}$ and since $f$ is continuous, we get $B^{*}(\beta)=f\left(A^{*}(\beta)\right)$ compact convex, which means that $B^{*}(\beta)$ is a closed interval for any $\beta \in[0,1]$.

If $\beta_{1} \leq \beta_{2}$, then $A^{*}\left(\beta_{1}\right) \subseteq A^{*}\left(\beta_{2}\right)$. This implies

$$
B^{*}\left(\beta_{1}\right)=f\left(A^{*}\left(\beta_{1}\right)\right) \subseteq f\left(A^{*}\left(\beta_{2}\right)\right)=B^{*}\left(\beta_{2}\right) .
$$

Let $\beta_{n}$ be a sequence which converges from above to $\beta \in[0,1)$. Then

$$
\bigcap_{n=1}^{\infty} A^{*}\left(\beta_{n}\right)=A^{*}(\beta) .
$$

We will show that

$$
\bigcap_{n=1}^{\infty} B^{*}\left(\beta_{n}\right)=B^{*}(\beta),
$$

i.e.,

$$
\bigcap_{n=1}^{\infty} f\left(A^{*}\left(\beta_{n}\right)\right)=f\left(A^{*}(\beta)\right),
$$

which is equivalent to

$$
\bigcap_{n=1}^{\infty} f\left(A^{*}\left(\beta_{n}\right)\right)=f\left(\bigcap_{n=1}^{\infty} A^{*}\left(\beta_{n}\right)\right) .
$$

Let

$$
y \in f\left(\bigcap_{n=1}^{\infty} A^{*}\left(\beta_{n}\right)\right) .
$$

Then, there exists $x \in A^{*}\left(\beta_{n}\right), \forall n \geq 1$, such that $y=f(x)$. Then $y \in f\left(A^{*}\left(\beta_{n}\right)\right), \forall n \geq 1$, i.e.,

$$
y \in \bigcap_{n=1}^{\infty} f\left(A^{*}\left(\beta_{n}\right)\right) \text {. }
$$


Now, let

$$
y \in \bigcap_{n=1}^{\infty} f\left(A^{*}\left(\beta_{n}\right)\right)
$$

Then $y \in f\left(A^{*}\left(\beta_{n}\right)\right), \forall n \geq 1$. Then, there exists an $x_{n} \in A^{*}\left(\beta_{n}\right)$ with $y=f\left(x_{n}\right), \forall n \geq 1$. Since $\left\{x_{n}\right\}$ is a bounded sequence and since $x_{n} \in A^{*}(1)$ and $A^{*}(1)$ is compact, it has a convergent subsequence $\left\{x_{n_{k}}\right\}$. Let $x=\lim _{k \rightarrow \infty} x_{n_{k}}$. Now, we will prove that $x \in A^{*}(\beta)$. Since

$$
\bigcap_{k=1}^{\infty} A^{*}\left(\beta_{n_{k}}\right)=A^{*}(\beta)
$$

we have $\lim _{k \rightarrow \infty} A_{1}^{*}\left(\beta_{n_{k}}\right)=A_{1}^{*}(\beta)$ and $\lim _{k \rightarrow \infty} A_{2}^{*}\left(\beta_{n_{k}}\right)=A_{2}^{*}(\beta)$. If $x \notin A^{*}(\beta)$, then there exists $K$ such that $x \notin A^{*}\left(\beta_{n_{k}}\right), \forall k \geq K$. Taking into account that $x=\lim _{k \rightarrow \infty} x_{n_{k}}$, we obtain a contradiction. Hence,

$$
\bigcap_{n=1}^{\infty} B^{*}\left(\beta_{n}\right)=B^{*}(\beta)
$$

Let $\left\{\beta_{n}\right\}$ be a sequence which converges from below to 1 . Then,

$$
c l\left(\bigcup_{n=1}^{\infty} A^{*}\left(\beta_{n}\right)\right)=A^{*}(1)
$$

Since $f$ is continuous, we have $f(c l(A)) \subseteq c l(f(A))$, and hence

$$
B^{*}(1)=f\left(\operatorname{cl}\left(\bigcup_{n=1}^{\infty} A^{*}\left(\beta_{n}\right)\right)\right) \subseteq \operatorname{cl}\left(\bigcup_{n=1}^{\infty} f\left(A^{*}\left(\beta_{n}\right)\right)\right)=c l\left(\bigcup_{n=1}^{\infty} B^{*}\left(\beta_{n}\right)\right)
$$

which implies

$$
B^{*}(1)=c l\left(\bigcup_{n=1}^{\infty} B^{*}\left(\beta_{n}\right)\right)
$$

Let us define the following relation

$$
\widetilde{A}^{i} \leq \widetilde{B}^{i} \Leftrightarrow A(\alpha) \subseteq B(\alpha) \text { and } B^{*}(\beta) \subseteq A^{*}(\beta)
$$

Corollary 3.2. Let $f: \mathbb{R} \rightarrow \mathbb{R}$ be a continuous function and $\tilde{f}^{i}: I F_{N}(\mathbb{R}) \rightarrow I F_{N}(\mathbb{R})$ be its intuitionistic fuzzy extension. If $\widetilde{A}^{i} \leq \widetilde{B}^{i}$, then $\widetilde{f}^{i}\left(\widetilde{A}^{i}\right) \leq \widetilde{f}^{i}\left(\widetilde{B}^{i}\right)$.

Proof. Let the $\alpha$ - and $\beta$-cuts of $\tilde{f}^{i}\left(\widetilde{A}^{i}\right)$ be $C(\alpha)$ and $C^{*}(\beta)$, respectively and the $\alpha$ - and $\beta$-cuts of $\widetilde{f}^{i}\left(\widetilde{B}^{i}\right)$ be $D(\alpha)$ and $D^{*}(\beta)$, respectively. By Theorem 3.1 we have $C(\alpha)=\widetilde{f}^{i}(A(\alpha))$, $C^{*}(\beta)=\tilde{f}^{i}\left(A^{*}(\beta)\right)$ and $D(\alpha)=\tilde{f}^{i}(B(\alpha)), D^{*}(\beta)=\tilde{f}^{i}\left(B^{*}(\beta)\right)$.

Since $f$ is a continuous function and $\widetilde{A}^{i} \leq \widetilde{B}^{i}$ implies $A(\alpha) \subseteq B(\alpha)$ and $B^{*}(\beta) \subseteq A^{*}(\beta)$, we obtain $f(A(\alpha)) \subseteq f(B(\alpha))$ and $f\left(B^{*}(\beta)\right) \subseteq f\left(A^{*}(\beta)\right)$. By the intuitionistic fuzzy extension principle we obtain that $C(\alpha) \subseteq D(\alpha)$ and $D^{*}(\beta) \subseteq C^{*}(\beta)$. Hence $\widetilde{f}^{i}\left(\widetilde{A}^{i}\right) \leq \widetilde{f}^{i}\left(\widetilde{B}^{i}\right)$. 
Definition 3.3. Let $f: X \times Y \rightarrow Z$ be a function. It can be extended to $f: I F(X) \times I F(Y) \rightarrow I F(Z)$ such that $\tilde{C}^{i}=f\left(\tilde{A}^{i}, \tilde{B}^{i}\right)$, where

$$
\begin{aligned}
& \mu_{\tilde{C}^{i}}(z)=\left\{\begin{array}{cc}
\sup _{x \in X, y \in Y}\left\{\min \left\{\mu_{\tilde{A}^{i}}(x), \mu_{\tilde{B}^{i}}(y)\right\}: f(x, y)=z\right\} ; & z \in f(X \times Y) \\
0 ; & z \notin f(X \times Y)
\end{array},\right. \\
& \nu_{\tilde{C}^{i}}(z)=\left\{\begin{array}{cc}
\inf _{x \in X, y \in Y}\left\{\max \left\{\nu_{\tilde{A}^{i}}(x), \nu_{\tilde{B}^{i}}(y)\right\}: f(x, y)=z\right\} ; & z \in f(X \times Y) \\
1 ; & z \notin f(X \times Y)
\end{array} .\right.
\end{aligned}
$$

Theorem 3.4. Given a continuous function $f: \mathbb{R} \times \mathbb{R} \rightarrow \mathbb{R}$, it can be extended to an intuitionistic fuzzy function $\tilde{f}^{i}: I F_{N}(\mathbb{R}) \times I F_{N}(\mathbb{R}) \rightarrow I F_{N}(\mathbb{R})$ such that for $\tilde{A}^{i} \times \tilde{B}^{i} \in I F_{N}(\mathbb{R}) \times I F_{N}(\mathbb{R})$ we can determine $\tilde{C}^{i}=\tilde{f}^{i}\left(\tilde{A}^{i}, \tilde{B}^{i}\right) \in I F_{N}(\mathbb{R})$ by its $\alpha$ - and $\beta$-cuts $C(\alpha)=\tilde{f}^{i}(A(\alpha), B(\alpha))$ and $C^{*}(\beta)=\tilde{f}^{i}\left(A^{*}(\beta), B^{*}(\beta)\right), \forall \alpha, \beta \in[0,1]$, i.e., we have $C(\alpha)=\left[C_{1}(\alpha), C_{2}(\alpha)\right]$ and $\left.C^{*}(\beta)=\left[C_{1}^{*}(\beta), C_{2}^{*}(\beta)\right)\right]$, where

$$
\begin{aligned}
& C_{1}(\alpha)=\inf \{f(x, y):(x, y) \in(A(\alpha), B(\alpha))\}, \\
& C_{2}(\alpha)=\sup \{f(x, y):(x, y) \in(A(\alpha), B(\alpha))\}, \\
& C_{1}^{*}(\beta)=\inf \left\{f(x, y):(x, y) \in\left(A^{*}(\beta), B^{*}(\beta)\right)\right\}, \\
& C_{2}^{*}(\beta)=\sup \left\{f(x, y):(x, y) \in\left(A^{*}(\beta), B^{*}(\beta)\right)\right\},
\end{aligned}
$$

$\forall \alpha, \beta \in[0,1]$

Proof. We will first prove that if $A(\alpha), A^{*}(\beta), B(\alpha), B^{*}(\beta)$, and $C(\alpha), C^{*}(\beta)$ are $\alpha$ - and $\beta$-cuts of the intuitionistic fuzzy numbers $\tilde{A}^{i}, \tilde{B}^{i}$, and $\tilde{C}^{i}=\tilde{f}^{i}\left(\tilde{A}^{i}, \tilde{B}^{i}\right)$, respectively, then $C(\alpha)=$ $\tilde{f}^{i}(A(\alpha), B(\alpha))$ and $C^{*}(\beta)=f\left(A^{*}(\beta), B^{*}(\beta)\right)$. By [6], we know that $C(\alpha)=\tilde{f}^{i}(A(\alpha), B(\alpha))$. Let us prove that $C^{*}(\beta)=f\left(A^{*}(\beta), B^{*}(\beta)\right)$.

If $f^{-1}(z)=\emptyset$, then $\nu_{\tilde{C}^{i}}(z)=1$ and there is nothing to do. Assume that $f^{-1}(z) \neq \emptyset$. Then, by Definition 3.3, we have

$$
\nu_{\tilde{C}^{i}}(z)=\inf _{x \in X, y \in Y}\left\{\max \left\{\nu_{\tilde{A}^{i}}(x), \nu_{\tilde{B}^{i}}(y)\right\}: f(x, y)=z\right\} .
$$

If $x \in A^{*}(\beta)$ and $y \in B^{*}(\beta)$, then $\nu_{\tilde{A}^{i}}(x) \leq \beta$ and $\nu_{\tilde{B}^{i}}(y) \leq \beta$ and it implies also that $\nu_{\tilde{C}^{i}}(z) \leq \beta$ and so $z=f(x, y) \in C^{*}(\beta)$, i.e., $f\left(A^{*}(\beta), B^{*}(\beta)\right) \subseteq C^{*}(\beta)$. On the other hand, if $\nu_{\tilde{C}^{i}}(z) \leq \beta$, then for each $\varepsilon=\frac{1}{n}, n \in \mathbb{N}$, there exists an $\left(x_{n}, y_{n}\right) \in f^{-1}(z)$ such that

$$
\nu_{\tilde{A}^{i}}\left(x_{n}\right)<\nu_{\tilde{C}^{i}}(z)+\frac{1}{n}
$$

and

$$
\nu_{\tilde{B}^{i}}\left(y_{n}\right)<\nu_{\tilde{C}^{i}}(z)+\frac{1}{n} .
$$

Let $\nu_{\tilde{A}^{i}}\left(x_{n}\right) \leq \beta_{n}$ and $\nu_{\tilde{B}^{i}}\left(y_{n}\right) \leq \beta_{n}$. Since $\left\{x_{n}\right\}$ is a bounded sequence and since $x_{n} \in A^{*}(1)$ with $A^{*}(1)$ is compact, it has a convergent subsequence $\left\{x_{n_{k}}\right\}$. Let $x=\lim _{k \rightarrow \infty} x_{n_{k}}$. Since $\nu_{\tilde{A}^{i}}$ is lower-semi continuous, it follows that $\lim _{k \rightarrow \infty} \nu_{\tilde{A}^{i}}\left(x_{n_{k}}\right) \geq \nu_{\tilde{A}^{i}}(x)$. Hence, from (2) and the fact that $\nu_{\tilde{C}^{i}}(z) \leq \beta$, we have $\nu_{\tilde{A}^{i}}(x) \leq \beta$ also. Similarly, since $\left\{y_{n}\right\}$ is a bounded sequence and $y_{n} \in$ 
$B^{*}(1)$ and $B^{*}(1)$ is compact, it has a convergent subsequence $\left\{y_{n_{k}}\right\}$. Let $y=\lim _{k \rightarrow \infty} y_{n_{k}}$. Since $\nu_{\tilde{B}^{i}}$ is lower-semi continuous, it follows that $\lim _{k \rightarrow \infty} \nu_{\tilde{B}^{i}}\left(y_{n_{k}}\right) \geq \nu_{\tilde{B}^{i}}(y)$. Hence, from (3) and the fact that $\nu_{\tilde{C}^{i}}(z) \leq \beta$, we have $\nu_{\tilde{B}^{i}}(y) \leq \beta$ also. And since $f\left(x_{n_{k}}, y_{n_{k}}\right)=z$ and $f$ is continuous, $f(x, y)=z$. Then, $C^{*}(\beta) \subseteq f\left(A^{*}(\beta), B^{*}(\beta)\right)$. Finally, we have $C^{*}(\beta)=f\left(A^{*}(\beta), B^{*}(\beta)\right)$. So, if $\left.C^{*}(\beta)=\left[C_{1}^{*}(\beta), C_{2}^{*}(\beta)\right)\right]$, we obtain

$$
\begin{aligned}
& C_{1}^{*}(\beta)=\inf \left\{f(x, y):(x, y) \in\left(A^{*}(\beta), B^{*}(\beta)\right)\right\}, \\
& C_{2}^{*}(\beta)=\sup \left\{f(x, y):(x, y) \in\left(A^{*}(\beta), B^{*}(\beta)\right)\right\} .
\end{aligned}
$$

From now on, the proof is similar to that of the preceding theorem. Since $\left.A^{*}(\beta), B^{*}(\beta)\right)$ are compact convex intervals in $\mathbb{R}$ and since $f$ is continuous, $C^{*}(\beta)=f\left(A^{*}(\beta), B^{*}(\beta)\right)$ is compact and convex.

If $\beta_{1} \leq \beta_{2}$, then $A^{*}\left(\beta_{1}\right) \subseteq A^{*}\left(\beta_{2}\right)$ and $B^{*}\left(\beta_{1}\right) \subseteq B^{*}\left(\beta_{2}\right)$, which implies that

$$
C^{*}\left(\beta_{1}\right)=f\left(A^{*}\left(\beta_{1}\right), B^{*}\left(\beta_{1}\right)\right) \subseteq f\left(A^{*}\left(\beta_{2}\right), B^{*}\left(\beta_{2}\right)\right)=C^{*}\left(\beta_{2}\right) .
$$

Let $\left\{\beta_{n}\right\}$ be a sequence which converges from above to $\beta \in[0,1)$, we have

$$
\bigcap_{n=1}^{\infty} C^{*}\left(\beta_{n}\right)=C^{*}(\beta),
$$

which can be shown similarly as in the previous theorem. Finally, if $\left\{\beta_{n}\right\}$ is a sequence which converges from below to 1 , we obtain

$$
C^{*}(1)=c l\left(\bigcup_{n=1}^{\infty} C^{*}\left(\beta_{n}\right)\right) .
$$

\section{Conclusion}

In Theorem 3.1 we have proved that the $\alpha$ - and $\beta$-cuts of the image of an intuitionistically fuzzified crisp function obtained by the intuitionistic fuzzy Zadeh's extension principle coincide with the $\alpha$ - and $\beta$-cuts of an intuitionistic fuzzy number. With the help of this result we have shown that for any intuitionistic fuzzy numbers $\widetilde{A}^{i}$ and $\widetilde{B}^{i}$ with $\widetilde{A}^{i} \leq \widetilde{B}^{i}$ the inequality $\tilde{f}^{i}\left(\widetilde{A}^{i}\right) \leq \widetilde{f}^{i}\left(\widetilde{B}^{i}\right)$ holds. After giving Definition 3.3 we have extended the results in Theorem 3.1 to

$$
I F_{N}(\mathbb{R}) \times I F_{N}(\mathbb{R})
$$

\section{References}

[1] Akın, Ö., \& Bayeğ, S. (2017). Intuitionistic fuzzy initial value problems: An application. Hacettepe Journal of Mathematics and Statistics, 48(6), 1682-1694.

[2] Akın, Ö., \& Bayeğ, S. (2019). Some results on the fundamental concepts of fuzzy set theory in intuitionistic fuzzy environment by using $\alpha$ - and $\beta$-cuts. Filomat, 33(10), 3123-3148. 
[3] Atanassov, K. (1986). Intuitionistic fuzzy sets. Fuzzy Sets and Systems, 20(1), 87-96.

[4] Atanassova, L. (2006). On Intuitionistic fuzzy versions of L. Zadeh's extension principle. Notes on Intuitionistic Fuzzy Sets, 13(3), 33-36.

[5] Dong, W. M., \& Wong, F. S. (1987). Fuzzy weighted averages and implementation of the extension principle. Fuzzy Sets and Systems, 21(2), 183-199.

[6] De Barros, L. C., Bassanezi, R. C., \& Tonelli, P.A. (1997). On the continuity of the Zadeh's extension. 7th IFSA World Congress Proceedings, Prague, Czech Republic, 3-8.

[7] Hanss, M. (2002). The transformation method for the simulation and analysis of systems with uncertain parameters. Fuzzy Sets and Systems, 130(3), 277-289.

[8] Kerre, E. E. (2011). A tribute to Zadeh's extension principle. Scientia Iranica, 18(3), 593-595.

[9] Marinov, E. (2014). On extension principle for intuitionistic fuzzy sets. Notes on Intuitionistic Fuzzy Sets, 20(3), 34-41.

[10] Nguyen, H. T. (1978). A note on the extension principle for fuzzy sets. Journal of Mathematical Analysis and Applications, 64(2), 369-380.

[11] Wood, K. L., Otto, K. N., \& Antonsson, E. K. (1992). Engineering design calculations with fuzzy parameters. Fuzzy Sets and Systems, 52(1), 1-20. 